\title{
Evaluación de los micromovimientos en la interfase hueso-implante con carga inmediata: análisis de elementos finitos
}

\author{
Ronald Oswaldo Velásquez-Paz, Est., Esp.. ${ }_{1}$ Samuel David Giraldo-Gómez ${ }^{*}$, \\ Esp. $_{1}$, Federico Latorre-Correa, Esp..$_{2}$, Junes Abdul Villarraga-Ossa, MSc. ${ }_{3}$, \\ Juan Gustavo Diosa-Peña, Est., PhD. ${ }_{3}$
}

Facultad de Odontología, Universidad Cooperativa de Colombia

${ }_{2}$ Facultad de Odontología, Universidad de Antioquia

${ }_{3}$ Facultad de Ingeniería, Universidad de Antioquia

Recibido: 11 de enero del 2017 Aprobado: 27 de febrero del 2017

*Autor de correspondencia: Samuel David Giraldo Gómez. Calle 37 Sur, Envigado, Antioquia. Teléfono: (4) 4446065 Correo electrónico: samuel.giraldo@campusucc.edu.co

Cómo citar este artículo: Velásquez-Paz RO, Giraldo-Gómez SD, Latorre-Correa F, Villarraga-Ossa JA, Diosa-Peña JG. Evaluación de los micromovimientos en la interfase hueso-implante con carga inmediata: análisis de elementos finitos. Rev Nac Odontol. 2017;13(25):57-65. doi: http://dx.doi.org/10.16925/od.v13i25.1882

Resumen. Introducción: el propósito de esta investigación fue evaluar los micromovimientos en la interfase hueso-implante utilizando un pilar provisional de poliéter cetona (PEEK) y una corona de polimetilmetacrilato (PMMA) sometidos a carga inmediata, con la finalidad de establecer si estos micromovimientos pueden perjudicar la oseointegración del implante bajo cargas normales. Esta evaluación fue realizada a través del método de análisis de elementos finitos (MEF). Métodos: se modeló un implante Tapered Screw-Vent ${ }^{\circledR}$ (ref. Tsvi10 Zimmer Dental) de $13 \mathrm{~mm}$ de longitud por 3,7 $\mathrm{mm}$ de diámetro con una plataforma de 3,5 $\mathrm{mm}$, un pilar de poliéter cetona, un tornillo, una corona de polimetilmetacrilato de un incisivo central superior, y se modelaron un hueso cortical y un hueso esponjoso utilizando el software de diseño asistido por computadora (CAD) de Solid Works 2010 (Solid Works Corp., Concord, Massachussets, Estados Unidos), y después se procesó y analizó a través del software ANsys versión 14. Se evaluaron los micromovimientos en la interfase hueso-implante aplicando cargas en sentido oblicuo con una magnitud de 200 Newtons en la superficie palatina del incisivo central superior. Resultados: el hueso esponjoso y el cortical presentaron micromovimientos con valores similares $(31,57$ y $32,88 \mu \mathrm{m}$, respectivamente). Conclusiones: los micromovimientos máximos se dieron a nivel del cuello del implante. Sin embargo, el hueso de alta densidad está preparado para recibir implantes con carga inmediata sin poner en riesgo el proceso de oseointegración.

Palabras clave: análisis de elementos finitos, implante dental, interfase huesoimplante, micromovimientos. 


\title{
Evaluation of micromovements at the bone-implant interface with immediate loading: Analysis of finite elements
}

\begin{abstract}
Introduction: The purpose of this research was to evaluate micromovements at the bone-implant interface using a provisional polyetheretherketone (PEEK) abutment and a polymethyl methacrylate (PMMA) crown subjected to immediate loading, in order to establish whether these micromovements can impair implant osseointegration under normal loads. This evaluation was carried out through the finite element analysis (FEM) method. Methods: A $13 \mathrm{mmL}, 3.7 \mathrm{mmD}$ Tapered Screw-Vent ${ }^{\oplus}$ implant (ref. (tsvb10 Zimmer Dental) was modeled with a $3.5 \mathrm{~mm}$ platform, a PEEK abutment, a screw, and a PMMA crown of an upper central incisor. A cortical bone and a cancellous bone were modeled using Solid Works 2010 computer-aided design (CAD) software (Solid Works Corp., Concord, Massachusetts, United States), and then processed and analyzed by the ANSYs 14.0 software. Micromovements at the bone-implant interface were evaluated by applying loads obliquely with a force of 200 Newtons on the palatal surface of the upper central incisor. Results: The cancellous and cortical bones showed micromovements with similar values (31.57 and $32.88 \mu \mathrm{m}$, respectively). Conclusions: The maximum micromovements occurred at the level of the implant neck. However, the high-density bone is prepared to receive implants with immediate loading without jeopardizing the osseointegration process.
\end{abstract}

Keywords: finite element analysis, dental implant, bone-implant interface, micromovements.

\section{Avaliação dos micromovimentos na interfase osso-implante com carga imediata: análise de elementos finitos}

Resumo. Introdução: o propósito desta pesquisa foi avaliar os micromovimentos na interfase osso-implante utilizando um pilar provisório de polietercetona (PEEK) e uma coroa dentária de polimetilmetacrilato (PMMA) submetidos a carga imediata, com a finalidade de estabelecer se esses micromovimentos podem prejudicar a osseointegração do implante sob cargas normais. Essa avaliação foi realizada por meio do método de análise de elementos finitos (MEF). Métodos: modelou-se implante Tapered Screw-Vent ${ }^{\oplus}$ (ref. Tsvi10 Zimmer Dental) de $13 \mathrm{~mm}$ de longitude por 3,7 $\mathrm{mm}$ de diâmetro com uma plataforma de 3,5 $\mathrm{mm}$, um pilar de PEEK, um parafuso, uma coroa dentária de PMMA de um incisivo central superior, e modelaram-se um osso cortical e um osso esponjoso utilizando o software de desenho assistido por computador (CAD) de Solid Works 2010 (Solid Works Corp., Concord, Massachussets, Estados Unidos); em seguida, processou-se e analisou-se por meio do software ANSYs versão 14. Avaliaram-se os micromovimentos na interfase osso-implante aplicando cargas em sentido oblíquo com uma magnitude de 200 Newtons na superfície palatina do incisivo central superior. Resultados: o osso esponjoso e o cortical apresentaram micromovimentos com valores similares (31,57 e 32,88 $\mu \mathrm{m}$, respectivamente). Conclusões: os micromovimentos máximos foram dados no nível do colo do implante. Contudo, o osso de alta densidade está preparado para receber implantes com carga imediata sem colocar em risco o processo de osseointegração.

Palavras-chave: análise de elementos finitos, implante dental, interfase osso-implante, micromovimentos. 


\section{Introducción}

El protocolo de carga inmediata tiene como finalidad la colocación de una corona provisional inmediatamente después de la inserción del implante. Actualmente, es predecible realizar dicho protocolo gracias a los cambios geométricos y superficiales de los implantes, así como a la comprensión de los procesos fisiológicos de cicatrización que mejoran la estabilidad primaria y aceleran el proceso de oseointegración. Hay varios puntos de vista con respecto a realizar o no la carga inmediata, pero la decisión se basa en diferentes criterios que van desde el tipo de implante que se colocó, hasta las diferentes condiciones que se pueden encontrar en el paciente, entre ellas la cantidad de hueso disponible (amplitud, espesor y altura) y la calidad o densidad de este (hueso tipo I, II, III o IV) [1]. Múltiples estudios prospectivos y revisiones sistemáticas han soportado la evidencia acerca de los implantes colocados bajo el protocolo de carga inmediata $[2,3]$.

Fisiológicamente, el ligamento periodontal posee la capacidad de absorber los esfuerzos, lo que resulta en cierto grado de movilidad dental. Becker y Kaiser [4] reportaron que un diente natural sano posee micromovimientos de 50 a $200 \mu \mathrm{m}$. Gross [5] reportó que un implante oseointegrado posee micromovimientos normales de 10 a $50 \mu \mathrm{m}$, básicamente por las propiedades elásticas del hueso. Sin embargo, en etapas tempranas de la cicatrización, el micromovimiento puede ser perjudicial, porque conduce a la formación de una capa de tejido conectivo fibroso adyacente al implante, evitando la oseointegración. Brunski [6] reportó que micromovimientos más allá de $100 \mu \mathrm{m}(0,1 \mathrm{~mm})$ deben evitarse para favorecer la oseointegración temprana.

En el protocolo de tratamiento con implantes, está indicada la utilización de pilares provisionales previo a los definitivos, en un periodo variable que puede extenderse por varios meses según sean la necesidad y los objetivos terapéuticos de cada caso. Actualmente, los pilares provisionales son elaborados en PEEK o titanio. El titanio es reconocido por su alto módulo de elasticidad y excelente biocompatibilidad; sin embargo, su coloración puede ocasionar problemas estéticos [7]. El PEEK posee un módulo de elasticidad muy cercano al del hueso, lo que favorece una adecuada distribución de esfuerzos y promueve una remodelación ósea óptima [8].
Esta investigación tuvo el propósito de analizar los micromovimientos en la interfase hueso-implante, utilizando un pilar provisional en PEEK y corona de PMMA bajo carga inmediata. La investigación fue realizada in vitro a través del método de análisis de elementos finitos, ampliamente utilizado para predecir el comportamiento biomecánico de varios diseños de implantes dentales y el efecto de los factores clínicos en su éxito [9]. Dammak [10] y Tissakht et al. [11] concluyeron que los modelos de elementos finitos son una herramienta útil para predecir los micromovimientos en la interfase hueso-implante. Viceconti et al. [12] realizaron un estudio comparativo entre modelos experimentales de hueso femoral y modelos generados mediante el método de elementos finitos, concluyendo que estos últimos tienen la precisión necesaria para predecir la estabilidad primaria de un implante y si los micromovimientos pueden o no dañar la respectiva oseointegración.

\section{Materiales y métodos}

Se elaboró el diseño de un modelo geométrico tridimensional, simulando una restauración protésica provisional implantosoportada, con pilar de PEEK y corona de PMMA sometidas a carga inmediata mediante el software CAD de Solid Works 2010 (Solid Works Corp., Concord, Massachussets, Estados Unidos). A continuación, se presentan las características de cada uno de los componentes modelados:

- Un implante Tapered Screw-Vent ${ }^{\circledR}$ de $13 \mathrm{~mm}$ de longitud x 3,7 mm de diámetro, con una plataforma de 3,5 mm de hexágono interno con su respectivo tornillo de fijación. Referencia número: Tsvi10, Zimmer ${ }^{\circledR}$ Dental 1900 Aston Avenue Carlsbad, California 92008-7308 Estados Unidos.

- Un pilar de PEeK Zimmer ${ }^{\circledR}$, recto, de conexión interna, plataforma de $3,5 \mathrm{~mm}$, perfil de emergencia de $4,5 \mathrm{~mm}$, altura de margen vestibular de $1 \mathrm{~mm}$. Referencia número: PT341S, Zimmer ${ }^{\circledR}$ Dental 1900 Aston Avenue Carlsbad, California 92008-7308 Estados Unidos.

- Una corona de PMma.

- Hueso cortical y hueso esponjoso.

Se escogió un implante para un incisivo central superior, así como su respectivo pilar con el 
diámetro y la conexión más utilizadas para la rehabilitación oral en el sector anterior [13]. Todas las estructuras se modelaron de forma individual.

También, se modeló una sección de hueso tipo D II, según la clasificación de Lekholm y Zarb [1], reproducida a partir de un corte sagital del maxilar superior, extraído de una imagen tomográfica, incluyendo hueso cortical y hueso esponjoso. Desde el punto de vista mecánico, el hueso es considerado anisotrópico porque muestra distintas propiedades mecánicas dependiendo de la dirección desde la cual sean medidas [14]. Sin embargo, a causa de la complejidad de la modelación de una estructura con dichas características usando el método de elementos finitos, puede ser aplicable una aproximación según el concepto de isotropía transversal, teniendo en cuenta que el módulo elástico del hueso medido en ciertos ejes posee valores similares [14]. Por lo tanto, se evaluaron las propiedades isotrópicas para el implante, el pilar, el tornillo y la corona, y las propiedades transversalmente isotrópicas para el hueso cortical y el hueso esponjoso. En los modelos, no fue tomada en cuenta la encía asumiendo que no tiene un efecto significativo en cuanto a los micromovimientos en la interfase hueso-implante (figuras 1 y 2 ).

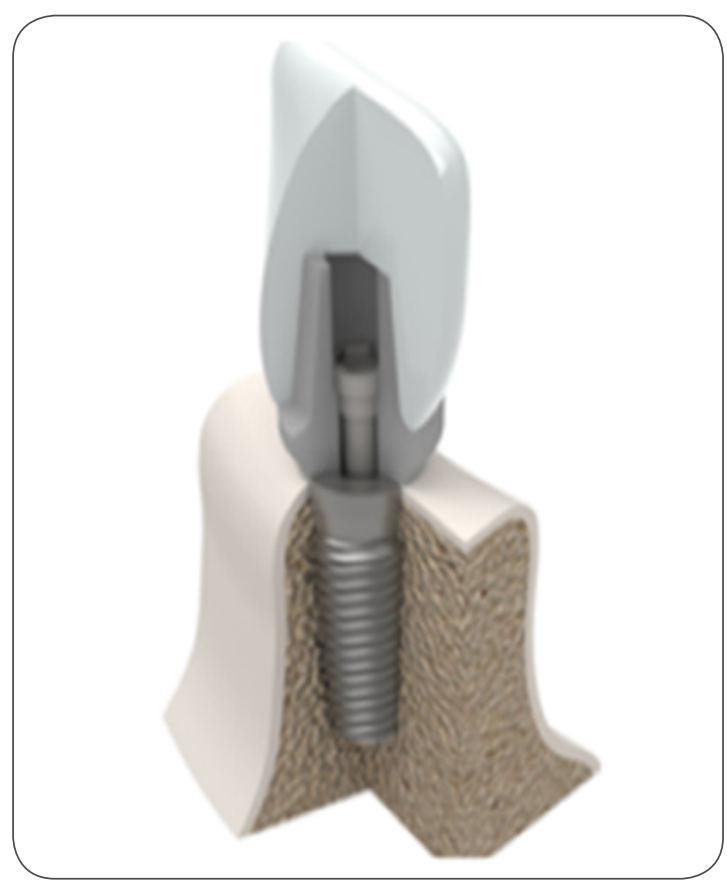

Figura 1. Imagen renderizada de hueso, implante, pilar, tornillo y corona

Fuente: elaboración propia

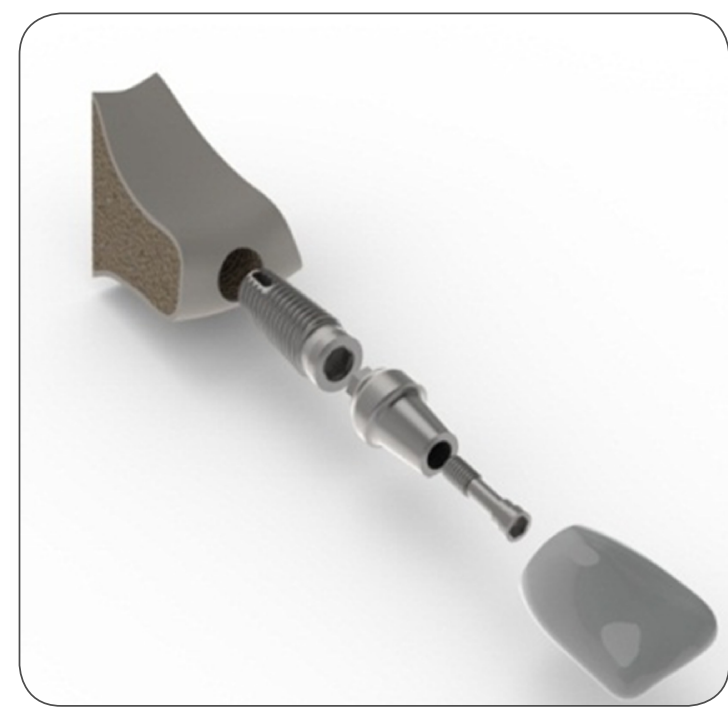

Figura 2. Imagen renderizada de hueso, implante, pilar, tornillo y corona, en la que se observa por separado cada componente

Fuente: elaboración propia

La condición de precarga en el tornillo se realizó mediante la aplicación de una carga térmica, empleando una fórmula matemática $(\mathrm{T}=\mathrm{KFd})$ sobre las roscas del tornillo, donde $T$ es igual al torque, $K$ es igual a $0,2, F$ es la fuerza axial y $d$ es igual al diámetro del tornillo. Esa contracción térmica uniaxial acorta el área entre las roscas, creando la precarga en el tornillo y generando una fuerza entre este y el implante sin disminuir el diámetro original del implante. La cantidad de torque del tornillo fue de $30 \mathrm{~N} \cdot \mathrm{cm}$ según lo recomendado por la casa fabricante $[15,16]$.

Para determinar el comportamiento mecánico de la estructura, se realizó un análisis elástico lineal en el que se utilizaron elementos tetraédricos y algunos hexaédricos, con el fin de obtener una mejor aproximación de las geometrías de los componentes. De esta manera, se obtuvo una malla tridimensional de cada uno de los componentes del modelo (figura 3 y tabla 1 ).

Al validar las características de la malla, se observó alta calidad de los elementos $(0,8198)$. Para mejorar la precisión, se utilizó el método adaptativo $\mathrm{H}$, el cual consiste en realizar un refinamiento en el tamaño de la malla en los lugares de mayor interés para el estudio. En este caso, la zona en donde ocurren los mayores desplazamientos o esfuerzos [17]. 


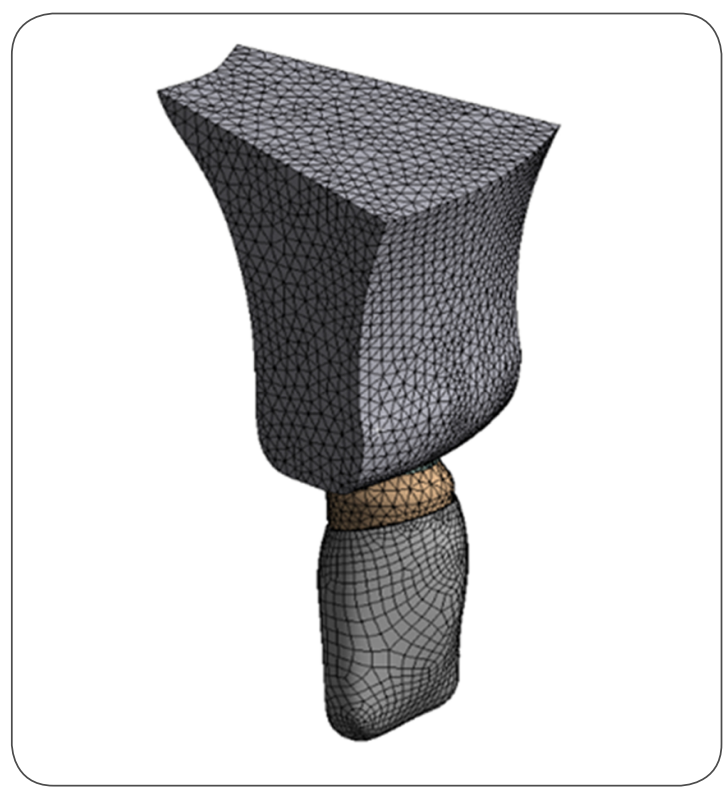

Figura 3. Malla sólida tetraédrica de las estructuras Fuente: elaboración propia
Para evaluar los micromovimientos mediante el modelo, se utilizaron las constantes elásticas: i) el modelo elástico de Young, que es la propiedad de los materiales que equivale a la pendiente de la curva de esfuerzo contra la deformación en el rango elástico; ii) la razón de Poisson, que es una constante elástica de los materiales que indica la relación entre la deformación axial y lateral; y iii) el límite de fluencia, que es la deformación irrecuperable o irreversible para cada uno de los elementos que se van a analizar. Las propiedades mecánicas de los materiales a usar en este estudio se muestran en la tabla 2 .

Una vez obtenida la modelación, se realizó un análisis de los micromovimientos en la interfase hueso-implante a través del software ANsys versión 14. Se aplicó una carga de $200 \mathrm{~N}$, que corresponde a la carga oclusal normal promedio reportada por la literatura para el sector anterior en un incisivo central superior [22], con un vector de fuerza oblicuo a una angulación de $45^{\circ}$ sobre la cara palatina del

Tabla 1. Detalles de la malla de los modelos estudiados

\begin{tabular}{cccc}
\hline Pieza & Número de nodos & Número de elementos & Tipo de elemento \\
\hline Corona & 26334 & 8369 & Hexaedros \\
Tornillo & 102681 & 68304 & Tetraedros \\
Pilar & 32753 & 21865 & Tetraedros \\
Implante & 771900 & 522533 & Tetraedros \\
Hueso cortical & 1256481 & 875139 & Tetraedros \\
Hueso esponjoso & 1125139 & 789348 & Tetraedros \\
\hline
\end{tabular}

Fuente: elaboración propia

Tabla 2. Propiedades mecánicas de las estructuras y los materiales modelados

\begin{tabular}{|c|c|c|c|c|c|c|c|}
\hline \multirow{2}{*}{ Material } & \multicolumn{3}{|c|}{ Límite elástico $(\mathrm{MPa})$} & \multicolumn{3}{|c|}{ Coeficiente de Poisson } & \multirow{2}{*}{$\begin{array}{l}\text { Límite de fluencia } \\
(\mathrm{MPa})\end{array}$} \\
\hline & $\mathrm{X}$ & $\mathrm{Y}$ & $\mathrm{Z}$ & $\mathbf{x x}$ & $\mathbf{X Y}$ & $\mathbf{x z}$ & \\
\hline Hueso cortical [14] & 10,000 & 11,000 & 14,300 & 370 & 330 & 230 & $60-120$ \\
\hline Hueso esponjoso [14] & 1148 & 210 & 1148 &, 50 &, 320 &, 10 & $60-120$ \\
\hline Implante de titanio $[18,19]$ & 110,000 & 110,000 & 110,000 &, 330 &, 330 & ,330 & 800 \\
\hline Tornillo del pilar titanio [15] & 110,000 & 110,000 & 110,000 & ,330 &, 330 &, 330 & 800 \\
\hline Pilar de PEeK [20] & 4,300 & 4,300 & 4,300 &, 37 &, 37 &, 37 & 172 \\
\hline Corona de pмма $[14,21]$ & 3,000 & 3,000 & 3,000 & ,38 & ,38 & ,38 & 72 \\
\hline
\end{tabular}

Fuente: elaboración propia 
diente rehabilitado, en un área de $4 \mathrm{~mm}^{2}$. De esta manera, fue posible evaluar el comportamiento a nivel de la interfase (figura 4).

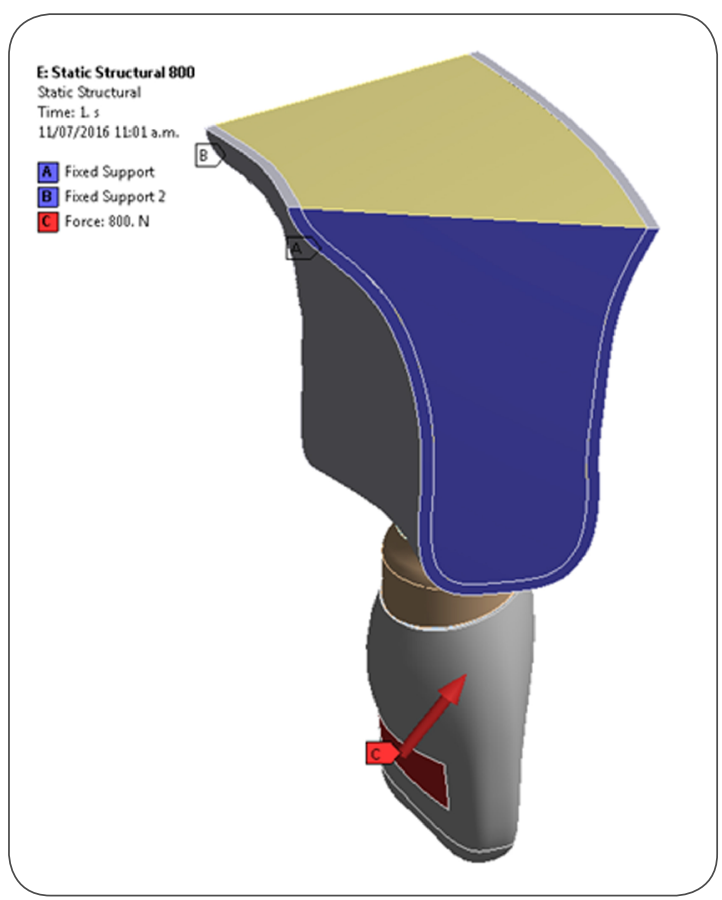

Figura 4. Dirección y ubicación de las cargas (áreas en rojo) en el modelo

Fuente: elaboración propia

\section{Resultados}

Al someter el modelo a una carga de $200 \mathrm{~N}$, los micromovimientos en el hueso esponjoso mostraron valores de 3,5 a $31,57 \mu \mathrm{m}$. Por su parte, los micromovimientos en el hueso cortical mostraron valores entre 3,65 y 32,88 $\mu \mathrm{m}$. Los valores más altos fueron registrados en la tabla vestibular del hueso, a nivel de la zona crestal (figuras 5 y 6 ).

\section{Discusión}

La estabilidad primaria del implante es esencial para que se dé el proceso de oseointegración, dado que el éxito del tratamiento está más relacionado con la función crítica de los micromovimientos en la interfase hueso-implante que con el tiempo de carga [23].

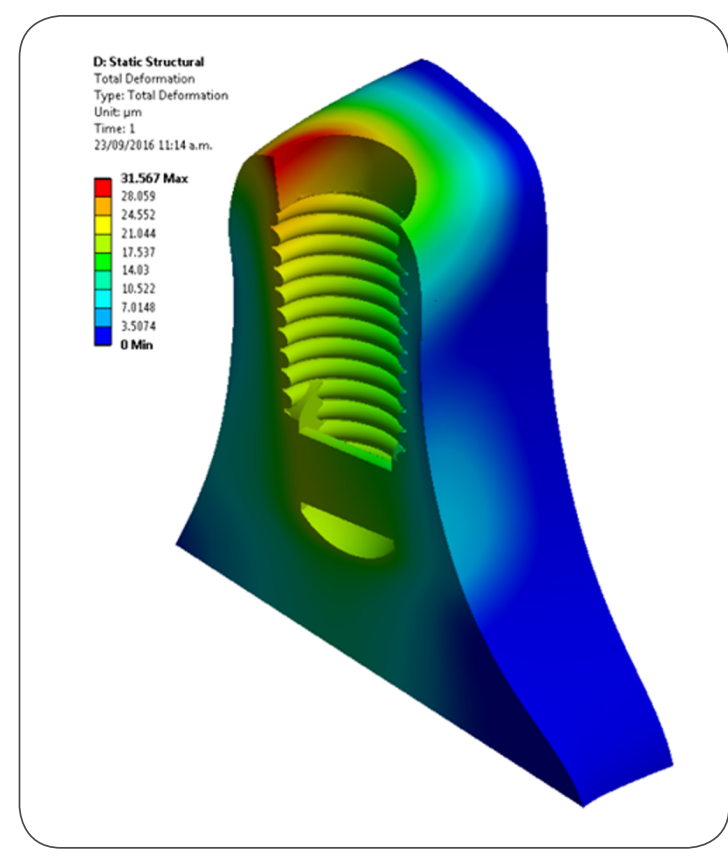

Figura 5. Distribución de los micromovimientos en el hueso esponjoso con $200 \mathrm{~N}$ de carga sobre un pilar de PEEK y una corona de PMMA.

Fuente: elaboración propia

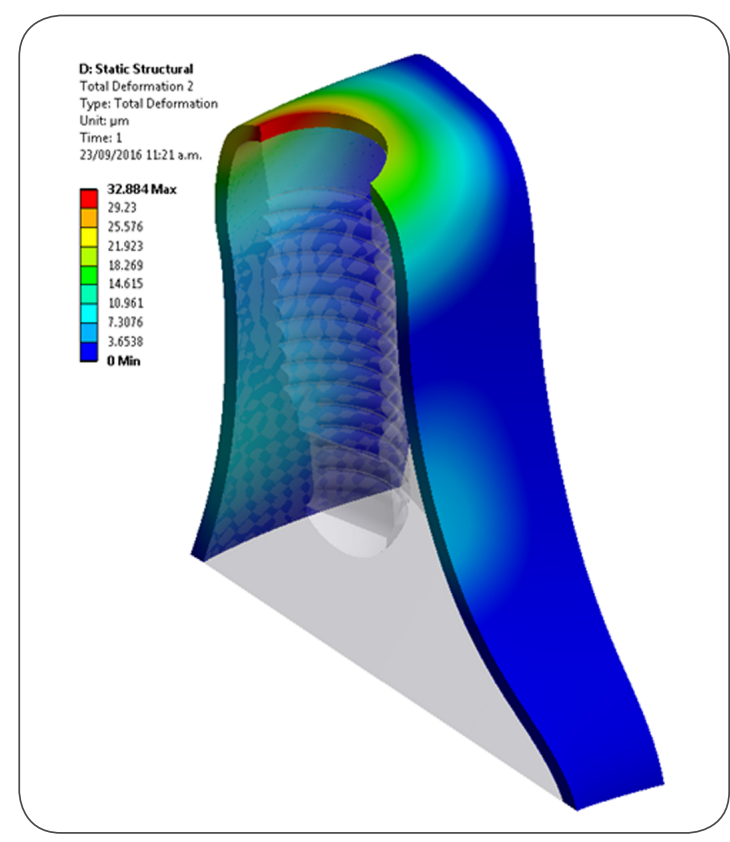

Figura 6. Distribución de los micromovimientos en el hueso cortical con $200 \mathrm{~N}$ de carga sobre un pilar de PEEK y una corona de PMma.

Fuente: elaboración propia 
Los implantes con superficies texturizadas han sido diseñados para que se dé la fijación mediante el crecimiento óseo a ese nivel, pero hay evidencia clínica de que este fenómeno no siempre ocurre. Szmukler [24] definió dos conceptos de movimiento en la interfase hueso-implante: por un lado, los micromovimientos son aquellos que permiten la formación de hueso sobre la superficie del implante, y por otro, los macromovimientos conducen a la formación de tejido conectivo fibroso y se encuentran en el rango de 150 a $500 \mu \mathrm{m}$.

Vandamme et al. [25] realizaron un experimento in vitro y reportaron que micromovimientos entre 30 y $90 \mu \mathrm{m}$ estimulan la neoformación ósea en la superficie del implante. En el presente estudio, los micromovimientos encontrados para la interfase hueso-implante tanto en el hueso cortical como esponjoso mostraron valores similares, cercanos a $32 \mu \mathrm{m}$, compatibles con la zona de estimulación ósea reportada por Vandamme et al. [25]. Sin embargo, micromovimientos de 100 a $150 \mu \mathrm{m}$ son el límite máximo, previo a causar inhibición del crecimiento óseo. En otro estudio, Soballe [26] reportó que el movimiento controlado de los implantes en el rango de $500 \mu \mathrm{m}$ inhibe la oseointegración, independientemente del tipo de textura superficial del implante. Pilliar [27] presentó un estudio en el cual concluyó que movimientos de $150 \mu \mathrm{m}$ o más estimulan la formación de tejido fibroso maduro en lugar de hueso.

Aunque la densidad del hueso esponjoso es importante para la generación de micromovimientos, el hueso cortical puede ser un factor crítico para evitar que estos sean excesivos en implantes cargados inmediatamente, ya que estudios llevados a cabo con el método de elementos finitos han demostrado que cuando se aplican fuerzas laterales u oblicuas sobre el implante, la mayor magnitud de estas fuerzas se concentra en el hueso cortical. Por tal motivo, varios estudios se han orientado más a evaluar la influencia del espesor del hueso cortical que la densidad ósea [23].

Sugiura [23] realizó un estudio de elementos finitos con el objetivo de establecer la influencia de la densidad del hueso y el espesor del cortical en relación con los micromovimientos de la interfase hueso-implante, y reportó que en modelos de hueso esponjoso de alta densidad el implante se desplazó más a nivel coronal que apical, con valores por debajo de $30 \mu \mathrm{m}$, mientras que en modelos de hueso esponjoso de baja densidad los micromovimientos fueron aproximadamente de $100 \mu \mathrm{m}$. Este hallazgo muestra que los micromovimientos se van incrementando proporcionalmente a la disminución de la densidad del hueso esponjoso, por lo cual es un factor crítico en implantes cargados inmediatamente. El espesor del hueso cortical juega un rol importante en la reducción de los micromovimientos solo cuando el hueso esponjoso posee baja densidad.

El estudio de Sugiura [23] también reportó micromovimientos máximos de $17 \mu \mathrm{m}$ en un hueso cortical de $2 \mathrm{~mm}$ de espesor. Esto muestra un comportamiento similar al reportado en el presente estudio, en el que los micromovimientos fueron aproximadamente dos veces mayores, pero con un espesor de hueso cortical de $1 \mathrm{~mm}$. Huang et al. [28] reportaron que el micromovimiento máximo en un modelo de hueso esponjoso de alta densidad fue de $7,9 \mu \mathrm{m}$, bajo una carga oblicua de $129 \mathrm{~N}$ a $30^{\circ}$.

Se debe tomar en cuenta que la presente investigación es una simulación in vitro, que expone información teórica sobre el comportamiento de los elementos modelados, por lo que no se debe extrapolar directamente al ambiente clínico sin las investigaciones complementarias del caso.

\section{Conclusiones}

- Los micromovimientos máximos se dieron a nivel del cuello del implante $(32,88 \mu \mathrm{m})$. Sin embargo, los valores obtenidos ofrecen un amplio margen de seguridad para dar lugar a la oseointegración.

- Los micromovimientos a nivel de hueso cortical y esponjoso mostraron valores similares, tomando en cuenta que los dos son de alta densidad.

- El hueso de alta densidad está preparado para recibir implantes con carga inmediata sin poner en riesgo el proceso de oseointegración.

\section{Conflicto de interés}

Los autores no tienen conflictos de interés de ningún tipo para la elaboración y la presentación de resultados del presente artículo. 


\section{Referencias}

[1] Lekholm U, Zarb GA. Patient selection. Tissue-integrated prostheses: Osseointegration in clinical dentistry. Chicago: Quintessence Publishing; 1985.

[2] Strub JR, Jurdzik BA, Tuna T. Prognosis of immediately loaded implants and their restorations: A systematic literature review. J Oral Rehabil. 2012;39(9):704-17. doi: 10.1111/j.1365-2842.2012.0 2315.x

[3] Testori T, Meltzer A, Del Fabbro M, Zuffetti F, Troiano M, Francetti L, et al. Immediate occlusal loading of Osseotite implants in the lower edentulous jaw. A multicenter prospective study. Clin Oral Implants Res. 2004;15(3):278-84. doi: 10.1111/j.16000501.2004.01013.x

[4] Becker CM, Kaiser DA, Jones JD. Guidelines for splinting implants. J Prosthet Dent. 2000; 84(2):2104. doi: http://doi.org/10.1067/mpr.2000.108672.

[5] Gross MD. Occlusion in implant dentistry. A review of the literature of prosthetic determinants and current concepts. Aust Dent J. 2008;53:(1 Suppl):S60 -S68. doi: 10.1111/j.1834-7819.2008.00043.x

[6] Brunsky J. Avoid pitfalls of overloading and micromotion of intraosseous implants. Dent Implantol Update. 1993;4(10):76-81.

[7] Peixoto HE, Bordin D, Del Bel Cury AA, Da Silva WJ, Faot F. The role of prosthetic abutment material on the stress distribution in a maxillary single implant-supported fixed prosthesis. Mater Sci Eng C. 2016;65:90-6.

[8] Najeeb S, Zafar MS, Khurshid Z, Siddiqui F. Applications of polyetheretherketone (PEEK) in oral implantology and prosthodontics. J Prosthodont Res. 2016;60(1):12-9. doi: http://doi.org/10.1016/j.jpor. 2015.10.001

[9] Hasan I, Röger B, Heinemann F, Keilig L, Bourauel C. Influence of abutment design on the success of immediately loaded dental implants: Experimental and numerical studies. Med Eng Phys. 2012;34(7): 817-25. doi: http://doi.org/10.1016/j.medengphy. 2011.09 .023

[10] Dammak M, Shirazi-Adl A, Zukor DJ. Analysis of cementless implants using interface nonlinear friction - Experimental and finite element studies. J Biomech. 1997;30(2):121-9. doi: https://doi.org/10. 1016/S0021-9290(96)00110-8

[11] Tissakht M, Eskandari H, Ahmed AM. Micromotion analysis of the fixation of total knee tibial component. Computers \& Structures. 1995;56(2-3):365-75. doi: https://doi.org/10.1016/0045-7949(95)00029-G

[12] Vicenconti M, Muccini R, Bernakiewicz M, Baliani M, Cristofolini L. Large-sliding contact ele- ments accurately predict levels of bone-implant micromotion relevant to osseointegration. J Biomech. 2000;33(12):1611-8

[13] Roldán FM, Escobar JC, Latorre F, Villarraga J. Análisis lineal y no lineal de los esfuerzos en pilares de circonio preformados. Elementos finitos. Revista Ingeniería Biomédica. 2014;8(16):39-50

[14] Kurniawan D, Nor FM, Lee HY, Lim JY. Finite element analysis of bone-implant biomechanics: Refinement through featuring various osseointegration conditions. Int J Oral Maxillofac Surg. 2012;41 (9):1090-6

[15] Alkan I, Sertgöz A, Ekici B. Influence of occlusal forces on stress distribution in preloaded dental implant screws. J Prosthodontic Dentistry. 2004; 91(4):319-25. doi: http://doi.org/10.1016/j.prosdent. 2004.01.016

[16] Jaroslow B, Gazis D. A multi-attachment approach to partial dentures. Trends Tech Contemp Dent Lab. 1985;2(10):39-44.

[17] Fuentemayor F, Payares F. Definición de malla óptima en refinamiento $\mathrm{H}$-Adaptativo para múltiples casos de carga. Mecánica Computacional. 2002;21: 3060-75.

[18] Niinomi N. Mechanical properties of biomedical titanium alloys. Mater Sci Eng A. 1998;243(1-2):231-6. doi: http://doi.org/10.1016/S0921-5093(97)00806-X

[19] Lin D, Li Q, Li W, Swain M. Dental implant induced bone remodeling and associated algorithms. J Mechbehav Biomed Mat. 2009;2(5):410-32. doi: http:// doi.org/10.1016/j.jmbbm.2008.11.007

[20] Schwitalla AD, Abou-Emara M, Spintig T, Lackmann J, Müller WD. Finite element analysis of the biomechanical effects of PEEK dental implants on the peri-implant bone. J Biomech. 2015;48(1):1-7. doi: http://doi.org/10.1016/j.jbiomech.2014.11.017

[21] Ridwan-Pramana A, Marcián P, Borák L, Narra N, Forouzanfar T, Wolff J. Structural and mechanical implications of PMMA implant shape and interface geometry in cranioplasty - A finite element study. J Craniomaxillofac Surg. 2016;44(1):34-44. doi: http:// doi.org/10.1016/j.jcms.2015.10.014

[22] O’Mahony AM, Williams JL, Spencer P. Anisotropic elasticity of cortical and cancellous bone in the posterior mandible increases peri-implant stress under oblique loading. Clin Oral Implants Res. 2001;12(6):648-57. doi: 10.1034/j.1600-0501. 2001. 120614.x

[23] Sugiura T, Yamamoto K, Horita S, Tsutsumi S, Kirita $\mathrm{T}$. The effects of bone density and crestal cortical bone thickness on micromotion and peri-implant bone strain distribution in an immediately loaded implant: A nonlinear finite element analysis. 
J Periodontal Implant Sci. 2016;46(3):152-65. doi: https://doi.org/10.5051/jpis.2016;46(3):152-65.

[24] Szmukler-Moncler S, Salama H, Reingewirtz Y, Dubruille JH. Timing of loading and effect of micromotion on bone-dental implant interface: Review of experimental literature. J Biomed Mater Res. 1998;43(2):192-203.

[25] Vandamme K, Naert I, Geris L, Vander Sloten J, Puers R, Duyck J. The effect of micromotion on the tissue response around immediately loaded roughened titanium implants in the rabbit. Eur J Oral Sci. 2007;115(1):21-9. doi: 10.1111/j.1600-0722.2007. 00 416.x
[26] Søballe K, Hansen ES, B-Rasmussen H, Jørgensen $\mathrm{PH}$, Bünger $\mathrm{C}$. Tissue ingrowth into titanium and hydroxyapatite-coated implants during stable and unstable mechanical conditions. J Orthop Res. 1992; 10(2):285-99. doi: 10.1002/jor.1100100216

[27] Pillar RM, Lee JM, Maniatopoulos C. Observations on the effect of movement on bone ingrowth into porous surfaced implant. Clin Orthop Relat Res. 1986;(208):108-13.

[28] Huang HL, Fuh LJ, Hsu JT, Tu MG, Shen YW, Wu CL. Effects of implant surface roughness and stiffness of grafted bone on an immediately loaded maxillary implant: A 3D numerical analysis. J Oral Rehabil. 2008;35(4):283-90. doi: 10.1111/j.1365-28 42.2007.01817.x 